IFT-P.000/2000

\title{
Cohomology in the Pure Spinor Formalism for the Superstring
}

\author{
Nathan Berkovits] \\ Instituto de Física Teórica, Universidade Estadual Paulista \\ Rua Pamplona 145, 01405-900, São Paulo, SP, Brasil
}

\begin{abstract}
A manifestly super-Poincaré covariant formalism for the superstring has recently been constructed using a pure spinor variable. Unlike the covariant Green-Schwarz formalism, this new formalism is easily quantized with a BRST operator and tree-level scattering amplitudes have been evaluated in a manifestly covariant manner.

In this paper, the cohomology of the BRST operator in the pure spinor formalism is shown to give the usual light-cone Green-Schwarz spectrum. Although the BRST operator does not directly involve the Virasoro constraint, this constraint emerges after expressing the pure spinor variable in terms of $\mathrm{SO}(8)$ variables.
\end{abstract}

June 2000

1 e-mail: nberkovi@ift.unesp.br 


\section{Introduction}

Ever since the light-cone Green-Schwarz (GS) superstring formalism was constructed [1], physicists have searched for a manifestly covariant version of the formalism. Such a formalism would have the advantage over the Ramond-Neveu-Schwarz formalism that scattering amplitudes could be computed in a manifestly super-Poincaré covariant manner. Although there exists a classical covariant GS description of the superstring [2] quantization problems have prevented this description from being used to compute scattering amplitudes. Recently, a new super-Poincaré covariant formalism for the superstring was constructed using pure spinor worldsheet variables in addition to the usual GS variables. Unlike all other covariant versions of the GS superstring, this pure spinor formalism is easy to quantize and was used to compute spacetime-supersymmetric tree amplitudes involving an arbitrary number of external massless states [3] [4].

Physical states in this new formalism are defined as states in the cohomology of the nilpotent operator

$$
Q=\int d \sigma \lambda^{\alpha}(z) d_{\alpha}(z)
$$

where $\lambda^{\alpha}$ is the pure spinor variable and $d_{\alpha}$ is the worldsheet variable for the spacetimesupersymmetric derivative [5]. Although it is easy to check that the massless states in the cohomology of $Q$ are those of ten-dimensional super-Yang-Mills [3] [6], it is a bit mysterious how the correct massive spectrum can be obtained since $Q$ does not directly involve the Virasoro constraint. In this paper, this mystery will be resolved and it will be shown that the cohomology of $Q$ indeed reproduces the desired light-cone GS spectrum.

As will be discussed in section 2, the first step in resolving the mystery is to express the pure spinor variable $\lambda^{\alpha}$ in terms of $\mathrm{SO}(8)$ variables. The pure spinor constraint $\lambda \gamma^{\mu} \lambda=0$ implies that $\left(\gamma^{+} \lambda\right)^{a}=s^{a}$ and $\left(\gamma^{-} \lambda\right)^{\dot{a}}=\sigma_{j}^{a \dot{a}} s^{a} v^{j}$ where $s^{a}$ is a null $\mathrm{SO}(8)$ spinor satisfying $s^{a} s^{a}=0$ and $v^{j}$ is an unconstrained $\mathrm{SO}(8)$ vector. In terms of $s^{a}$ and $v^{j}$,

$$
Q=\int d \sigma s^{a}\left[\left(\gamma^{-} d\right)^{a}+\sigma_{j}^{a \dot{a}} v^{j}\left(\gamma^{+} d\right)^{\dot{a}}\right]
$$

plus contributions from an infinite chain of ghosts-for-ghosts coming from the gauge invariance $\delta v^{j}=\sigma_{a \dot{a}}^{j} s^{a} \epsilon^{\dot{a}}$.

The second step in resolving the mystery is to enforce the first-class constraint $s^{a} s^{a}=0$ by modifying the BRST operator to

$$
Q^{\prime}=Q+\int d \sigma\left[-b s^{a} s^{a}+c\left(\frac{1}{2} \Pi^{-}+v^{j} \Pi^{j}+\frac{1}{2} v^{j} v^{j} \Pi^{+}\right)\right]
$$


where $(b, c)$ is the ghost and anti-ghost for the $s^{a} s^{a}$ constraint, $\Pi_{\mu}=\partial x_{\mu}-\frac{1}{2} \theta \gamma_{\mu} \partial \theta$ is the spacetime-supersymmetric momentum, and the term $c\left(\frac{1}{2} \Pi^{-}+v^{j} \Pi^{j}+\frac{1}{2} v^{j} v^{j} \Pi^{+}\right)$is required for nilpotency of $Q^{\prime}$. As will be argued in section 3, $Q^{\prime}$ has the same cohomology as $Q$ and is $\mathrm{SO}(9,1)$ super-Poincaré invariant.

Finally, it will be shown in section 4 that the cohomology of $Q^{\prime}$ reproduces the desired light-cone GS spectrum. Note that if one shifts $v^{j} \rightarrow v^{j}-\Pi^{j} / \Pi^{+}$in $Q^{\prime}$,

$$
Q^{\prime}=\int d \sigma\left[\left(\Pi^{+}\right)^{-1} s^{a}\left(\Pi_{\mu}\left(\gamma^{+} \gamma^{\mu} d\right)^{a}+\ldots\right)-b s^{a} s^{a}+\left(\Pi^{+}\right)^{-1} c\left(-\frac{1}{2} \Pi^{\mu} \Pi_{\mu}+\ldots\right)\right] .
$$

One can recognize $\Pi_{\mu} \gamma^{+} \gamma^{\mu} d$ as the first-class part of the GS fermionic constraints and $-\frac{1}{2} \Pi^{\mu} \Pi_{\mu}$ as the GS Virasoro constraint. The dependence of $Q^{\prime}$ on $v^{j}$ and the infinite chain of ghosts-for-ghosts is responsible for imposing the second-class part of the GS fermionic constraints. This use of an infinite set of fields for imposing second-class constraints resembles the treatment of chiral bosons in [7] and self-dual four-forms in [8].

\section{Construction of $Q$ using $\mathrm{SO}(8)$ Variables}

\subsection{Review of massless cohomology}

Physical states in the pure spinor formalism of the superstring are defined as ghostnumber one states in the cohomology of

$$
Q=\int d \sigma \lambda^{\alpha}(z) d_{\alpha}(z)
$$

where

$$
d_{\alpha}=p_{\alpha}+\frac{1}{2} \gamma_{\alpha \beta}^{\mu} \partial x_{\mu} \theta^{\beta}+\frac{1}{8} \gamma_{\alpha \beta}^{\mu} \gamma_{\mu \gamma \delta} \theta^{\beta} \theta^{\gamma} \partial \theta^{\delta}
$$

is the worldsheet variable for the supersymmetric derivative [5], $p_{\alpha}$ is the conjugate momentum to $\theta^{\alpha}$, and $\lambda^{\alpha}$ is a worldsheet variable carrying +1 ghost number and satisfying the pure spinor constraint

$$
\lambda^{\alpha}(z) \gamma_{\alpha \beta}^{\mu} \lambda^{\beta}(z)=0
$$

for $\mu=0$ to 9 . Since $d_{\alpha}(y) d_{\beta}(z) \rightarrow(y-z)^{-1} \gamma_{\alpha \beta}^{\mu} \Pi_{\mu}(z)$ where $\Pi^{\mu}=\partial x^{\mu}-\frac{1}{2} \theta^{\alpha} \gamma_{\alpha \beta}^{\mu} \partial \theta^{\beta}, Q$ is nilpotent.

To see that the open superstring 2 massless states are correctly reproduced by the cohomology of the zero modes of $Q$, recall that on-shell super-Yang-Mills can be described

2 Although only the open superstring will be discussed in this paper, all results are easily generalized to the heterotic and closed superstrings. 
by a spinor superfield $A_{\alpha}(x, \theta)$ satisfying $D_{\alpha}\left(\gamma^{\mu_{1} \ldots \mu_{5}}\right)^{\alpha \beta} A_{\beta}=0$ for any five-form direction $\mu_{1} \ldots \mu_{5}$ where $D_{\alpha}=\frac{\partial}{\partial \theta^{\alpha}}-\frac{1}{2} \theta^{\beta} \gamma_{\alpha \beta}^{\mu} \partial_{\mu}$ [9] [10] [6]. Using the gauge invariance $\delta A_{\alpha}=D_{\alpha} \Omega$, $A_{\alpha}$ can be gauge-fixed to

$$
A_{\alpha}(x, \theta)=a_{\mu}(x) \gamma_{\alpha \beta}^{\mu} \theta^{\beta}+\xi^{\gamma}(x) \gamma_{\alpha \beta}^{\mu} \gamma_{\mu \gamma \delta} \theta^{\beta} \theta^{\delta}+\ldots
$$

where $a_{\mu}(x)$ and $\xi^{\alpha}(x)$ are the linearized on-shell gluon and gluino of super-Yang-Mills and the component fields in ... are auxiliary fields which can be expressed in terms of $a_{\mu}$ and $\xi^{\gamma}$.

Since a massless vertex operator only depends on the worldsheet zero modes, $V=$ $\lambda^{\alpha} A_{\alpha}(x, \theta)$ for some $A_{\alpha}(x, \theta)$. But $Q V=0$ implies that $\lambda^{\alpha} \lambda^{\beta} D_{\alpha} A_{\beta}=0$, which can be decomposed into $\left(\lambda \gamma^{\mu} \lambda\right)\left(D \gamma_{\mu} A\right)+\left(\lambda \gamma^{\mu_{1} \ldots \mu_{5}} \lambda\right)\left(D \gamma_{\mu_{1} \ldots \mu_{5}} A\right)=0$. Since $\lambda \gamma^{\mu} \lambda=0, Q V=0$ implies the desired equation that $D \gamma_{\mu_{1} \ldots \mu_{5}} A=0$. Furthermore, the gauge invariance $\delta V=$ $Q \Omega=\lambda^{\alpha} D_{\alpha} \Omega$ reproduces the desired gauge transformation $\delta A_{\alpha}=D_{\alpha} \Omega$.

So the cohomology of the zero modes of $Q$ correctly reproduces on-shell super-YangMills. However, since $Q$ does not directly involve the Virasoro constraint, it is a bit mysterious how the mass-shell condition for the physical massive states is implied by $Q V=0$. As mentioned in the introduction, the first step to resolving this mystery is to express the pure spinor $\lambda^{\alpha}$ in terms of $\mathrm{SO}(8)$ representations.

\section{2. $S O(8)$ parameterization of a pure spinor}

An $\operatorname{SO}(9,1)$ spinor $\lambda^{\alpha}$ satisfying $\lambda \gamma^{\mu} \lambda=0$ contains eleven independent complex degrees of freedom. Together with their conjugate momenta, these eleven degrees of freedom contribute +22 to the central charge which cancels the sum of the central charge contributions of +10 from $x^{\mu}$ and -32 from $\left(\theta^{\alpha}, p_{\alpha}\right)$.

A convenient parameterization of $\lambda^{\alpha}$ is 3

$$
\lambda^{+}=\gamma, \quad \lambda_{A B}=\gamma u_{A B}, \quad \lambda^{A}=-\frac{1}{8} \gamma \epsilon^{A B C D E} u_{B C} u_{D E}
$$

where $A=1$ to $5, u_{A B}=-u_{B A}$ parameterizes the ten-dimensional complex space $S O(10) / U(5)$, and $\lambda^{\alpha}$ has been decomposed (after Wick rotation) into its $\mathrm{U}(5)$ components. However, since $\gamma$ is an overall scale parameter, this parameterization is singular when the $\lambda^{+}$component of $\lambda$ vanishes. Since physical states can exist with vanishing $\lambda^{+}$, the parameterization of (2.5) is inappropriate for computations of cohomology.3

3 For example, the massless vertex operator $V=\lambda^{\alpha} A_{\alpha}$ has physical degrees of freedom when $\lambda^{+}=0$. For this reason, the fact that $V=\left\{Q, \gamma^{-1} \theta^{+} V\right\}$ does not imply that $V$ is BRST-trivial since $\gamma^{-1} \theta^{+} V$ is not a well-defined operator. 
An alternative parameterization of $\lambda^{\alpha}$ is in terms of its $\operatorname{SO}(8)$ components $\left(\gamma^{+} \lambda\right)^{a}$ and $\left(\gamma^{-} \lambda\right)^{\dot{a}}$ where $\gamma^{ \pm}=\frac{1}{2}\left(\gamma^{0} \pm \gamma^{9}\right)$ and $(a, \dot{a})=1$ to 8 are chiral and anti-chiral $\operatorname{SO}(8)$ spinor indices. The constraint $\lambda \gamma^{-} \lambda=0$ implies that $s^{a}=\left(\gamma^{+} \lambda\right)^{a}$ satisfies $s^{a} s^{a}=0$. Furthermore, the constraint $\lambda \gamma^{j} \lambda=0$ implies that $\left(\gamma^{-} \lambda\right)^{\dot{a}}=\sigma_{j}^{a \dot{a}} v^{j} s^{a}$ for some $\mathrm{SO}(8)$ vector $v^{j}$ where $\sigma_{j}^{a \dot{a}}$ are the $\mathrm{SO}(8)$ Pauli matrices satisfying $\sigma_{(j}^{a \dot{a}} \sigma_{k)}^{b \dot{a}}=2 \delta_{j k} \delta^{a b}$. One can check that the constraint $\lambda \gamma^{+} \lambda=0$ implies no further conditions on $s^{a}$ and $v^{j}$. So the eleven degrees of freedome of $\lambda^{\alpha}$ can be parameterized by the seven degrees of freedom of a null spinor $s^{a}$ together with the eight degrees of freedom of $v^{j}$ as

$$
\left(\gamma^{+} \lambda\right)^{a}=s^{a}, \quad\left(\gamma^{-} \lambda\right)^{\dot{a}}=\sigma_{j}^{a \dot{a}} v^{j} s^{a}
$$

Unlike the $\mathrm{U}(5)$ parameterization of (2.5), this $\mathrm{SO}(8)$ parameterization is singular only when all eight components of $\left(\gamma^{+} \lambda\right)^{a}$ are zero. However, there are no physical states with vanishing $\left(\gamma^{+} \lambda\right)^{a}$, so the parameterization of (2.6) is appropriate for computing the cohomology

Since (2.6) is invariant under

$$
\delta v^{j}=\sigma_{a \dot{a}}^{j} s^{a} \epsilon^{\dot{a}}
$$

for arbitrary $\epsilon^{\dot{a}}$, this parameterization of $\lambda^{\alpha}$ has a gauge invariance which needs to be correctly treated. This can be done in the usual BRST manner by introducing a fermionic ghost $\mathrm{SO}(8)$ spinor variable $t^{\dot{a}}$. However, since $\delta \epsilon^{\dot{a}}=\sigma_{a \dot{a}}^{j} s^{a} y^{j}$ leaves the gauge transformation of (2.7) unchanged, one also needs to introduce a bosonic ghost-for-ghost $\mathrm{SO}(8)$ vector variable $v_{(1)}^{j}$. This line of reasoning continues ad infinitum to produce an infinite chain of bosonic $\mathrm{SO}(8)$ vectors, $v_{(0)}^{j}, v_{(1)}^{j}, \ldots$, and an infinite chain of fermionic $\mathrm{SO}(8)$ spinors, $t_{(0)}^{\dot{a}}, t_{(1)}^{\dot{a}}, \ldots$, where the original $v^{j}$ and $t^{\dot{a}}$ variables have been relabeled as $v_{(0)}^{j}$ and $t_{(0)}^{\dot{a}}$.

Since $s^{a}, v_{(n)}^{j}$ and $t_{(n)}^{\dot{a}}$ carry zero conformal weight, they contribute (together with their conjugate momenta) $+2(7+8-8+8-8+\ldots)$ to the central charge. Using the regularization familiar from $\kappa$-symmetry computations [11] that

$$
8-8+8-8+\ldots=\lim _{x \rightarrow 1} 8\left(1-x^{2}+x^{3}-x^{4}+\ldots\right)=\lim _{x \rightarrow 1} 8(1+x)^{-1}=4,
$$

4 For example, the gauge invariance $\delta A_{\alpha}=D_{\alpha} \Omega$ implies that one can choose the gauge $\left(\gamma^{+} A\right)^{\dot{a}}$ for the super-Yang-Mills spinor prepotential [10]. In this gauge, the massless vertex operator $V=\lambda^{\alpha} A_{\alpha}$ vanishes when $\left(\gamma^{+} \lambda\right)^{a}=0$. One expects that a similar gauge choice is possible for physical massive vertex operators such that they vanish when $\left(\gamma^{+} \lambda\right)^{a}=0$. 
one recovers the desired +22 contribution to the central charge.

Including the contribution of the ghost-for-ghosts, the BRST charge is $Q=\int d \sigma s^{a} G^{a}$ where

$$
G^{a}=\left(\gamma^{-} d\right)^{a}+\sigma_{j}^{a \dot{a}}\left[v_{(0)}^{j}\left(\gamma^{+} d\right)^{\dot{a}}+\sum_{n=0}^{\infty}\left(w_{(n)}^{j} t_{(n)}^{\dot{a}}+v_{(n+1)}^{j} u_{(n)}^{\dot{a}}\right)\right],
$$

$w_{(n)}^{j}$ is the conjugate momentum to $v_{(n)}^{j}$, and $u_{(n)}^{\dot{a}}$ is the conjugate momentum to $t_{(n)}^{\dot{a}}$. Note that $Q^{2}=0$ since $s^{a} s^{a}=0$ and $G^{a}(y) G^{b}(z) \rightarrow 2 \delta^{a b}(y-z)^{-1} T(z)$ where

$$
T=\frac{1}{2} \Pi^{-}+v^{j} \Pi^{j}+\frac{1}{2} v^{j} v^{j} \Pi^{+}+t_{(0)}^{\dot{a}}\left(\gamma^{+} d\right)^{\dot{a}}+\sum_{n=0}^{\infty}\left(v_{(n+1)}^{j} w_{(n)}^{j}+t_{(n+1)}^{\dot{a}} u_{(n)}^{\dot{a}}\right)
$$

and $\Pi^{ \pm}=\Pi^{0} \pm \Pi^{9}$.

Although $G^{a}(y) G^{b}(z) \rightarrow 2 \delta^{a b}(y-z)^{-1} T(z)$ suggests an $N=8$ super-Virasoro algebra, $G^{a}$ and $T$ are not super-Virasoro generators since, for example, $G^{a}$ and $T$ have +1 conformal weight and $T$ has no singular OPE's with either $G^{a}$ or $T$. Nevertheless, the resemblance with an $N=8$ algebra suggests that the BRST operator $Q$ can be modified to

$$
Q^{\prime}=\int d \sigma\left(s^{a} G^{a}+c T-b s^{a} s^{a}\right)
$$

where $(b, c)$ are fermionic ghosts of conformal weight $(1,0)$. It will be shown in the following section that $Q^{\prime}$ indeed has the same cohomology as $Q$.

\section{BRST treatment of the $s^{a} s^{a}=0$ Constraint}

\subsection{Equivalence of cohomology of $Q$ and $Q^{\prime}$}

Since the constraint $s^{a} s^{a}$ is included in the BRST operator $Q^{\prime}$ of (2.11), one expects that all eight components of $s^{a}$ can be treated as independent degrees of freedom in the 'off-shell' Hilbert space of $Q^{\prime}$. Note that this does not affect the central charge computation since the -2 contribution of the $(b, c)$ ghosts cancels the +2 contribution of the extra degree of freedom in $s^{a}$ and its conjugate momentum.

It will now be argued that the cohomology of $Q^{\prime}$ with $s^{a}$ unconstrained is equivalent to the cohomology of $Q$ with $s^{a}$ constrained to satisfy $s^{a} s^{a}=0$. Consider a state $V$ annihilated by $Q$ up to terms involving $s^{a} s^{a}$, i.e. $Q V=s^{a} s^{a} W$ for some $W$. Then $Q^{2}=s^{a} s^{a} T$ implies that $Q W=T V$. Using this information, one can check that the operator $V^{\prime}=V+c W$ is annihilated by $Q^{\prime}$. Furthermore, if $V$ is BRST-trivial up to terms 
involving $s^{a} s^{a}$, i.e. $V=Q \Omega+s^{a} s^{a} Y$ for some $Y$, then $V+c W=Q^{\prime}(\Omega-c Y)$ so $V^{\prime}$ is also BRST-trivial.

At the end of section 4, it will be shown that all physical states (with non-zero $\mathrm{P}^{+}$) in the cohomology of $Q^{\prime}$ can be written in the form $V^{\prime}=V+c W$ for some $V$ and $W$. Reversing the arguments of the previous paragraph, one learns that $V$ is in the cohomology of $Q$ up to terms involving $s^{a} s^{a}$. This proves equivalence of the cohomologies.

\subsection{Super-Poincaré invariance of $Q^{\prime}$}

Although $Q^{\prime}$ of (2.11) is expressed in terms of $\mathrm{SO}(8)$ variables, it will now be argued that $Q^{\prime}$ is invariant under $\mathrm{SO}(9,1)$ transformations. Since $Q^{\prime}$ is manifestly spacetimesupersymmetric, this implies the super-Poincaré invariance of $Q^{\prime}$. In terms of $\mathrm{SO}(8)$ representations, the pure spinor contribution to the $\mathrm{SO}(9,1)$ Lorentz currents is

$$
\begin{gathered}
N^{j k}=\frac{1}{2} s^{a}\left(\sigma^{j k}\right)_{a b} r^{b}+\sum_{n=0}^{\infty}\left[v_{(n)}^{[j} w_{(n)}^{k]}+\frac{1}{2} t_{(n)}^{\dot{a}}\left(\sigma^{j k}\right)_{\dot{a} \dot{b}} u_{(n)}^{\dot{b}}\right], \\
N^{j+}=w_{(0)}^{j}, \\
N^{+-}=b c-\frac{1}{2} s^{a} r^{a}+\sum_{n=0}^{\infty}\left[(n+1) v_{(n)}^{j} w_{(n)}^{j}+\left(n+\frac{3}{2}\right) t_{(n)}^{\dot{a}} w_{(n)}^{\dot{a}}\right], \\
N^{j-}=-3 \partial v_{(0)}^{j}-v_{(0)}^{k} N^{j k}-v_{(0)}^{j} N^{+-}-\frac{1}{2} v_{(0)}^{k} v_{(0)}^{k} w_{(0)}^{j}+v_{(0)}^{j} v_{(0)}^{k} w_{(0)}^{k}+\frac{1}{2} c \sigma_{a \dot{a}}^{j} t_{(0)}^{\dot{a}} r^{a}+F^{j-},
\end{gathered}
$$

where $r^{a}$ is the conjugate momentum to $s^{a}$ and it should be possible to determine the term $F^{j-}$ by requiring that

$\left[\int d \sigma N^{j-}, \sum_{n=0}^{\infty}\left(s^{a} \sigma_{j}^{a \dot{a}}\left(w_{(n)}^{j} t_{(n)}^{\dot{a}}+v_{(n+1)}^{j} u_{(n)}^{\dot{a}}\right)+c\left(v_{(n+1)}^{j} w_{(n)}^{j}+t_{(n+1)}^{\dot{a}} u_{(n)}^{\dot{a}}\right)\right)-s^{a} s^{a} b\right]=0$.

Note that $\left[s^{a}, \sigma_{j}^{a \dot{a}} v_{(0)}^{j} s^{a}+c t_{(0)}^{\dot{a}}\right]$ transform as the sixteen components of an $\mathrm{SO}(9,1)$ spinor and $\left[-\frac{1}{2}\left(c+c v_{(0)}^{k} v_{(0)}^{k}\right), c v_{(0)}^{j},-\frac{1}{2}\left(c-c v_{(0)}^{k} v_{(0)}^{k}\right)\right]$ transform as the ten components of an $\mathrm{SO}(9,1)$ vector, so the terms $\left[s^{a}\left(\gamma^{-} d\right)^{a}+\left(\sigma_{j}^{a \dot{a}} s^{a} v_{(0)}^{j}+c t_{(0)}^{\dot{a}}\right)\left(\gamma^{+} d\right)^{\dot{a}}\right]$ and $\left[\frac{1}{2} c \Pi^{-}+c v_{(0)}^{j} \Pi^{j}+\right.$ $\left.\frac{1}{2} c v_{(0)}^{k} v_{(0)}^{k} \Pi^{+}\right]$in $Q^{\prime}$ are easily seen to be Lorentz invariant.

Furthermore, one can check (up to the determination of $F^{j-}$ ) that $N^{\mu \nu}$ of (3.1) satisfies the OPE

$$
N^{\mu \nu}(y) N^{\rho \sigma}(z) \rightarrow \frac{\eta^{\rho[\nu} N^{\mu] \sigma}(z)-\eta^{\sigma[\nu} N^{\mu] \rho}(z)}{y-z}-3 \frac{\eta^{\mu \sigma} \eta^{\nu \rho}-\eta^{\mu \rho} \eta^{\nu \sigma}}{(y-z)^{2}}
$$


where the factor of 3 in the double pole comes from the pure spinor condition and is crucial for equivalence with the Lorentz generators in the Ramond-Neveu-Schwarz formalism for

the superstring [3]. For example, the double pole in $N^{j k}$ with $N^{j k}$ gets a contribution of +2 from the first term in $N^{j k}$ and a contribution of $+2-2+2-2+\ldots$ from the remaining terms. Using the regularization of [11],

$$
+2-2+2-2+\ldots=\lim _{x \rightarrow 1} 2(1+x)^{-1}=1
$$

so the total double pole contribution is +3 as desired. Simlilarly, the double pole of $N^{+-}$ with $N^{+-}$gets a contribution of +1 from the first term, -2 from the second term, and $-2\left(2^{2}-3^{2}+4^{2}-5^{2}+\ldots\right)$ from the remaining terms. This last expression can be regularized using the formula

$$
\sum_{n=0}^{\infty} n^{2}(-x)^{n}=2(1+x)^{-3}-3(1+x)^{-2}+(1+x)^{-1}
$$

which can be obtained by taking derivatives of the formula $\sum_{n=0}^{\infty}(-x)^{n}=(1+x)^{-1}$. So

$2^{2}-3^{2}+4^{2}-5^{2}+\ldots=1+\lim _{x \rightarrow 1} \sum_{n=0}^{\infty} n^{2}(-x)^{n}=1+\lim _{x \rightarrow 1}\left[2(1+x)^{-3}-3(1+x)^{-2}+(1+x)^{-1}\right]=1$

implying that the sum of the double pole contributions is -3 as desired.

So $Q^{\prime}$ has been shown to be a super-Poincaré invariant operator whose cohomology is equivalent to that of $Q=\int d \sigma \lambda^{\alpha} d_{\alpha}$. The cohomology of $Q^{\prime}$ will now be computed to be the light-cone GS spectrum.

\section{Evaluation of Cohomology of $Q^{\prime}$}

\subsection{Light-cone operators}

As mentioned earlier, $Q^{\prime}$ resembles the BRST operator for an $N=8$ super-Virasoro algebra. This can be made more evident by shifting $v_{(0)}^{j} \rightarrow v_{(0)}^{j}-\left(\Pi^{+}\right)^{-1} \Pi^{j}$ (where the zero mode of $\Pi^{+}$is assumed to be non-vanishing), so that $G^{a}=\left(\Pi^{+}\right)^{-1} \Pi_{\mu}\left(\gamma^{+} \gamma^{\mu} d\right)^{a}+\ldots$ and $T=-\frac{1}{2}\left(\Pi^{+}\right)^{-1} \Pi_{\mu} \Pi^{\mu}+\ldots$ The first term in $G^{a}$ can be recognized as the first-class part of the fermionic GS constraint and the first term in $T$ can be recognized as the GS Virasoro constraint. As will be explained below, the second-class part of the fermionic GS 
constraint will be implied by the infinite ghost-for-ghost dependence of $Q^{\prime}$ in a manner similar to the treatment of chiral bosons in [7] and self-dual four-forms in [8].

To compute the cohomology of $Q^{\prime}$, it is useful to first write $Q^{\prime}=Q_{1}+Q_{2}$ where

$$
Q_{1}=\int d \sigma\left[s^{a}\left(\gamma^{-} p\right)^{a}+\frac{1}{2} c\left(\partial x^{-}-P^{-}\right)\right], \quad Q_{2}=Q^{\prime}-Q_{1},
$$

and $P^{\mu}$ is the zero mode of $\partial x^{\mu}$. If $\left[\left(\gamma^{-} p\right)^{a},\left(\gamma^{+} \theta\right)^{a}\right]$ are assigned charge $(+1,-1),\left[\left(\partial x^{-}-\right.\right.$ $\left.\left.P^{-}\right),\left(\partial x^{-}-P^{+}\right)\right]$are assigned charge $(+1,-1)$, and all other variables are assigned zero charge, then $Q_{1}$ has charge +1 and all terms in $Q_{2}$ have non-positive charge.

So the cohomology of $Q^{\prime}$ is given by the cohomology of $Q_{2}$ restricted to operators in the cohomology of $Q_{1}$ [12]. But the cohomology of $Q_{1}$ consists of operators which are independent of $\left[\left(\gamma^{-} p\right)^{a},\left(\gamma^{+} \theta\right)^{a},\left(\partial x^{-}-P^{-}\right),\left(\partial x^{+}-P^{+}\right), s^{a}, r^{a}\right]$ and the non-zero modes of $(b, c)$. So the only term in $Q_{2}$ which survives in the cohomology of $Q_{1}$ is $c_{0}\left(T_{0}+\frac{1}{2} P^{-}\right)$ where

$$
\begin{gathered}
T_{0}=\int d \sigma\left[-\frac{1}{2} \theta \gamma^{-} \partial \theta+v_{(0)}^{j} \partial x^{j}+\frac{1}{2} v_{(0)}^{j} v_{(0)}^{j} P^{+}\right. \\
\left.+t_{(0)}^{\dot{a}}\left(\gamma^{+} p+\frac{1}{2} P^{+} \gamma^{-} \theta\right)^{\dot{a}}+\sum_{n=0}^{\infty}\left(v_{(n+1)}^{j} w_{(n)}^{j}+t_{(n+1)}^{\dot{a}} u_{(n)}^{\dot{a}}\right)\right] .
\end{gathered}
$$

A general operator in the cohomology of $Q_{1}$ can be written as $\mathcal{O}=A+b_{0} B+c_{0} C$ where $A, B, C$ are independent of $\left[\left(\gamma^{-} p\right)^{a},\left(\gamma^{+} \theta\right)^{a},\left(\partial x^{-}-P^{-}\right),\left(\partial x^{+}-P^{+}\right), s^{a}, r^{a}\right]$. Furthermore, $\left[Q_{2}, \mathcal{O}\right]=0$ implies that $\left[T_{0}+\frac{1}{2} P^{-}, A\right]=0$ and $B=0$. Finally, the gauge invariance $\delta \mathcal{O}=Q_{2} \Omega$ implies $\delta C=\left[T_{0}+\frac{1}{2} P^{-}, \Omega\right]$, so the cohomology associated with $C$ is related to that of $A$ by the usual doubling phenomenon associated with the $c_{0}$ ghost. So the cohomology of $Q^{\prime}$ can be evaluated by solving the equation $\left[T_{0}+\frac{1}{2} P^{-}, A\right]=0$.

Note that this same result can be obtained by using 'old covariant quantization' where one ignores the $\left(s^{a}, r^{a}\right)$ and $(c, b)$ ghosts. Using this method, one first uses $G^{a}$ and the nonzero modes of $T$ to gauge away $\left(\gamma^{+} \theta\right)^{a}$ and $\partial x^{+}$. Requiring that the operator $A$ commutes with $G^{a}$ and $T$ fixes $\left(\gamma^{-} p\right)^{a}$ and $\partial x^{-}$in terms of the remaining light-cone variables and implies that $\left[T_{0}+\frac{1}{2} P^{-}, A\right]=0$.

Since $T_{0}$ is quadratic in the remaining worldsheet variables, any operator $A$ satisfying $\left[T_{0}+\frac{1}{2} P^{-}, A\right]=0$ can be constructed from products of linear combinations of the variables, $a_{N}$, which satisfy $\left[T_{0}, a_{N}\right]=N a_{N}$ for some $N$. Then $\left[T_{0}+\frac{1}{2} P^{-}, A\right]=0$ implies the mass-shell condition that $-\frac{1}{2} P^{-}$is equal the sum of the eigenvalues in the product. For convenience, a Lorentz frame will be chosen where $P^{+}$is a non-zero fixed constant and $P^{j}=0$ for $j=1$ to 8 . 
One can easily check that $\left[T_{0}, y^{j}\right]=-\left(P^{+}\right)^{-1} \partial y^{j}$ and $\left[T_{0}, q^{\dot{a}}\right]=-\left(P^{+}\right)^{-1} \partial q^{\dot{a}}$ where

$$
y^{j}=\partial x^{j}+\sum_{n=0}^{\infty}\left(P^{+}\right)^{-n-1} \partial^{n+1} w_{(n)}^{j}, \quad q^{\dot{a}}=\left(\gamma^{+} p-\frac{1}{2} P^{+} \gamma^{-} \theta\right)^{\dot{a}}+\sum_{n=0}^{\infty}\left(P^{+}\right)^{-n-1} \partial^{n+1} u_{(n)}^{\dot{a}} .
$$

So the $M^{t h}$ mode of $y^{j}$ and $q^{\dot{a}}$ are eigenvectors of $T_{0}$ which carry eigenvalue $N=-M / P^{+}$. In fact, as will now be shown, these are the only normalizable eigenvectors of $T_{0}$ which can be constructed from linear combinations of the remaining variables.

First, suppose one has a bosonic eigenvector of $T_{0}$ of the form

$$
a_{N}=\int d \sigma\left[f_{N}^{j} \partial x^{j}+\sum_{n=0}^{\infty}\left(g_{N(n)}^{j} v_{(n)}^{j}+h_{N(n)}^{j} w_{(n)}^{j}\right)\right]
$$

where $\left(f_{N}^{j}, g_{N(n)}^{j}, h_{N(n)}^{j}\right)$ are coefficients of the eigenvector. Then $\left[T_{0}, a_{N}\right]=N a_{N}$ implies that

$$
\begin{aligned}
-h_{N(0)}^{j} & =N f_{N}^{j}, \quad-P^{+} h_{N(0)}^{j}-\partial f_{N}^{j}=N g_{N(0)}^{j}, \\
g_{N(n)}^{j} & =N g_{N(n+1)}^{j}, \quad-h_{N(n+1)}^{j}=N h_{N(n)}^{j} .
\end{aligned}
$$

Using the normalizability condition that $\int d \sigma\left[f_{N}^{j} f_{N}^{j}+\sum_{n=0}^{\infty} g_{N(n)}^{j} h_{N(n)}^{j}\right]$ is finite, one finds that the only normalizable solution of (4.5) is

$$
\partial f_{N}^{j}=P^{+} N f_{N}^{j}, \quad g_{N(n)}^{j}=0, \quad h_{N(n)}^{j}=(-N)^{n+1} f_{N}^{j},
$$

which is the $\left(-P^{+} N\right)^{t h}$ mode of the eigenvector $y^{j}$ of (4.3).

Second, suppose one has a fermionic eigenvector of $T_{0}$ of the form

$$
a_{N}=\int d \sigma\left[j_{N}^{\dot{a}}\left(\gamma^{+} p\right)^{\dot{a}}+k_{N}^{\dot{a}}\left(\gamma^{-} \theta\right)^{\dot{a}}+\sum_{n=0}^{\infty}\left(l_{N(n)}^{\dot{a}} t_{(n)}^{\dot{a}}+m_{N(n)}^{\dot{a}} u_{(n)}^{\dot{a}}\right)\right]
$$

where $\left(j_{N}^{\dot{a}}, k_{N}^{\dot{a}}, l_{N(n)}^{\dot{a}}, m_{N(n)}^{\dot{a}}\right)$ are coefficients of the eigenvector. Then $\left[T_{0}, a_{N}\right]=N a_{N}$ implies that

$$
\begin{gathered}
-m_{N(0)}^{\dot{a}}=N j_{N}^{\dot{a}}, \quad-\frac{1}{2} P^{+} m_{N(0)}^{\dot{a}}-\partial j_{N}^{\dot{a}}=N k_{N}^{\dot{a}}, \quad \frac{1}{2} P^{+} j_{N}^{\dot{a}}+k_{N}^{\dot{a}}=N l_{N(0)}^{\dot{a}}, \\
l_{N(n)}^{\dot{a}}=N l_{N(n+1)}^{\dot{a}}, \quad-m_{N(n+1)}^{\dot{a}}=N m_{N(n)}^{\dot{a}} .
\end{gathered}
$$

Using the normalizability condition that $\int d \sigma\left[j_{N}^{\dot{a}} k_{N}^{\dot{a}}+\sum_{n=0}^{\infty} l_{N(n)}^{\dot{a}} m_{N(n)}^{\dot{a}}\right]$ is finite, one finds that the only normalizable solution of (4.7) is

$$
\partial j_{N}^{\dot{a}}=P^{+} N j_{N}^{\dot{a}}, \quad k_{N}^{\dot{a}}=-\frac{1}{2} P^{+} j_{N}^{\dot{a}}, \quad l_{N(n)}^{\dot{a}}=0, \quad m_{N(n)}^{\dot{a}}=(-N)^{n+1} j_{N}^{\dot{a}},
$$

which is the $\left(-P^{+} N\right)^{t h}$ mode of the eigenvector $q^{\dot{a}}$ of (4.3).

So any operator satisfying $\left[T_{0}+\frac{1}{2} P^{-}, A\right]=0$ can be expressed as a product of the modes of $y^{j}$ and $q^{\dot{a}}$ multiplied by $e^{i P^{-} x^{+}}$where $\frac{1}{2} P^{+} P^{-}$is the sum of the mode numbers. By acting on a 'ground state' with non-zero $P^{+}$, these light-cone operators will now be used to construct physical states in the cohomology of $Q^{\prime}$. 


\subsection{Physical states}

Using the usual DDF construction [13], the light-cone operators $y^{j}$ and $q^{\dot{a}}$ of (4.3) can be extended to operators $\hat{y}^{j}$ and $\hat{q}^{\dot{a}}$ which commute with $G^{a}$ and $T$, and therefore commute with $Q^{\prime}$. Although $\hat{y}^{j}$ and $\hat{q}^{\dot{a}}$ will depend on the variables $\partial x^{+}$and $\left(\gamma^{+} \theta\right)^{a}$, they will be independent of the $(c, b)$ and $\left(s^{a}, r^{a}\right)$ ghosts. Any operator (with $P^{+}$non-zero and $\left.P^{j}=0\right)$ in the cohomology of $Q^{\prime}$ can be constructed from products of modes of $\hat{y}^{j}$ and $\hat{q}^{\dot{a}}$ multiplied by the appropriate factor of $e^{i P^{-} x^{+}}$.

Physical states in the cohomology of $Q^{\prime}$ are constructed by acting with these operators on a 'ground state' with non-zero $P^{+}$and $P^{j}=0$. Using the construction of section 3.1 together with the massless vertex operator of section 2.1, a suitable such ground state is

$$
V_{0}^{\prime}=\left(s^{a}\left[\left(\gamma^{-} A\right)^{a}+\sigma_{j}^{a \dot{a}} v_{(0)}^{j}\left(\gamma^{+} A\right)^{\dot{a}}\right]+c\left[\left(D \gamma^{-} A\right)+v_{(0)}^{j}\left(D \gamma^{j} A\right)+v_{(0)}^{j} v_{(0)}^{j}\left(D \gamma^{+} A\right)\right]\right) e^{i P^{+} x^{-}}
$$

where $A_{\alpha}$ is the on-shell super-Yang-Mills prepotential and $D_{\alpha}$ is the supersymmetric derivative. This state is annihilated by all negative modes of $\hat{y}^{j}$ and $\hat{q}^{\dot{a}}$, and the zero mode of $\hat{q}^{\dot{a}}$ acts as a spacetime supersymmetry transformation on $V_{0}^{\prime}$.

So the physical states in the cohomology of $Q^{\prime}$ (with non-zero $P^{+}$and $P^{j}=0$ ) can be represented by

$$
V^{\prime}=\prod_{j=1}^{8} \prod_{\dot{a}=1}^{8} \prod_{m, n=1}^{\infty}\left(\partial^{m} \hat{y}^{j}\right)^{\alpha_{m}^{j}}\left(\partial^{n} \hat{q}^{\dot{a}}\right)^{\beta_{n}^{\dot{a}}} e^{i P^{-} x^{+}} V_{0}^{\prime}
$$

where $\frac{1}{2} P^{+} P^{-}=\sum_{j, \dot{a}, m, n}\left(m \alpha_{m}^{j}+n \beta_{n}^{\dot{a}}\right)$. This is the usual light-cone GS spectrum. Note that all such states are of the form $V^{\prime}=V+c W$, which was needed in section 3.1 for proving equivalence of the $Q$ and $Q^{\prime}$ cohomologies.

Acknowledgements: I would like to especially thank Edward Witten for suggesting the comparison with light-cone Green-Schwarz, for his ideas concerning the cohomology computation, and for his collaboration during the initial stage of this work. I would also like to thank Cumrun Vafa for suggesting the importance of the $N=8$ algebra, Michael Bershadsky, Warren Siegel and Stefan Vandoren for useful conversations, CNPq grant 300256/94-9 for partial financial support, and the univerisities of Caltech, Harvard and SUNY at Stony Brook for their hospitality. This research was partially conducted during the period the author was employed by the Clay Mathematics Institute as a CMI Prize Fellow. 


\section{References}

[1] M.B. Green and J.H. Schwarz, Supersymmetrical Dual String Theory, Nucl. Phys. B181 (1981) 502.

[2] M.B. Green and J.H. Schwarz, Covariant Description of Superstrings, Phys. Lett. B136 (1984) 367.

[3] N. Berkovits, Super-Poincaré Covariant Quantization of the Superstring, JHEP 04 (2000) 018, hep-th/0001035.

[4] N. Berkovits and B.C. Vallilo, Consistency of Super-Poincaré Covariant Superstring Tree Amplitudes, hep-th/0004171.

[5] W. Siegel, Classical Superstring Mechanics, Nucl. Phys. B263 (1986) 93.

[6] P.S. Howe, Pure Spinor Lines in Superspace and Ten-Dimensional Supersymmetric Theories, Phys. Lett. B258 (1991) 141.

[7] B. McClain, Y.S. Wu and F. Yu, Covariant Quantization of Chiral Bosons and OSp(1,1/2) Symmetry, Nucl. Phys. B343 (1990) 689;

C. Wotzasek, The Wess-Zumino Term for Chiral Bosons, Phys. Rev. Lett. 66 (1991) 129.

[8] N. Berkovits, Manifest Electromagnetic Duality in Closed Superstring Field Theory, Phys. Lett. B388 (1996) 743, hep-th/9607070.

[9] W. Siegel, Superfields in Higher-Dimensional Spacetime, Phys. Lett. 80B (1979) 220;

B.E.W. Nilsson, Off-shell Fields for the Ten-Dimensional Supersymmetric Yang-Mills Theory, Gotenburg preprint 81-6 (Feb. 1981), unpublished;

B.E.W. Nilsson, Pure Spinors as Auxiliary Fields in the Ten-Dimensional Supersymmetric Yang-Mills Theory, Class. Quant. Grav. 3 (1986) L41.

[10] E. Witten, Twistor-like Transform in Ten Dimensions, Nucl. Phys. B266 (1986) 245.

[11] W. Siegel, Lorentz Covariant Gauges for Green-Schwarz Superstrings, talk at Strings '89, College Station Workshop (1989) 211;

S.J. Gates Jr., M.T. Grisaru, U. Lindstrom, M. Rocek, W. Siegel and P. van Nieuwenhuizen, Lorentz Covariant Quantization of the Heterotic Superstring, Phys. Lett. B225 (1989) 44;

R.E. Kallosh, Covariant Quantization of Type IIA,B Green-Schwarz Superstring, Phys. Lett. B225 (1989) 49;

M.B. Green and C.M. Hull, Covariant Quantum Mechanics of the Superstring, Phys. Lett. B225 (1989) 57.

[12] E. Witten, private communication.

[13] E. DelGiudice, P. DiVecchia and S. Fubini, General Properties of the Dual Resonance Model, Ann. Phys. 70 (1972) 378. 